\title{
Aspectos comportamentais do hospedeiro Diatraea saccharalis produzidos em
}

\section{laboratório}

\author{
Behavioral aspects of the host Diatraea saccharalis produced in a laboratory \\ Aspectos del comportamiento del hospedador Diatraea saccharalis producidos en un laboratorio
}

Recebido: 03/03/2021 | Revisado: 10/03/2021 | Aceito: 14/04/2021 | Publicado: 25/04/2021

Italo de Souza Aquino

ORCID: https://orcid.org/0000-0002-7948-8760 Universidade Federal da Paraíba, Brasil

italo.aquino@terra.com.br

Roberto Balbino da Silva

ORCID: https://orcid.org/0000-0003-4105-7858 Universidade Federal da Paraíba, Brasil balbinorobert@hotmail.com

Péricles de Farias Borges

ORCID: https://orcid.org/0000-0003-3585-1342 Universidade Federal da Paraíba, Brasil pericles@cca.ufpb.br

Alex da Silva Barbosa

ORCID: https://orcid.org/0000-0002-7343-6134 Universidade Federal da Paraíba, Brasil aldasibarbosa@cchsa.ufpb.br

Geovergue Rodrigues de Medeiros

ORCID: https://orcid.org/0000-0001-6544-1518 Instituto Nacional do Semiárido, Brasil geovergue.medeiros@insa.gov.br

\begin{abstract}
Resumo
A expansão no cultivo da cana-de-açúcar atrai vários insetos-praga, representando um fator limitante à cultura canavieira no Brasil. O objetivo da pesquisa foi analisar aspectos comportamentais, em laboratório, da broca da canade-açúcar Diatraea saccharalis como hospedeiro de Cotesia flavipes. O experimento foi desenvolvido em três etapas, de modo inteiramente casualizado. Na primeira etapa, correspondente à avaliação do ciclo biológico de vida ovo-adulto, o experimento utilizou 30 casais selecionados aleatoriamente da criação comercial do laboratório, sendo acomodados de modo individualizado. Na segunda etapa, realizou-se o diagnóstico da viabilidade de larva-pupa, larva-adulto e pupaadulto, bem como a razão sexual dos adultos de $D$. saccharalis. Foram coletadas 600 larvas durante cinco semanas (120 unidades por semana). Na terceira etapa, em relação às avaliações do experimento para mensurar a quantidade de larvas por casais, fez-se necessário adotar quatro tratamentos com 10 repetições cada um, sendo utilizado um casal adulto de inseto por câmara de acasalamento. Em todas as fases estudadas, os resultados destacam que a viabilidade desse hospedeiro apresenta índices acima de $90 \%$. Na razão sexual dos adultos, as fêmeas são maioria. Durante o período de oviposição, a quantidade média obtidas de larva por casal foi de 243 indivíduos. Conclui-se que a temperatura é um fator importante para prolongar ou diminuir o ciclo de vida da $D$. saccharalis.
\end{abstract}

Palavras-chave: Broca da cana-de-açúcar; Controle biológico; Produção de insetos benéficos.

\begin{abstract}
The expansion in the cultivation of sugar cane attracts several pest insects, representing a limiting factor to the sugarcane culture in Brazil. The objective of the research was to analyze the behavioral aspects, in laboratory, of the sugarcane borer Diatraea saccharalis as host of Cotesia flavipes. The experiment was developed in three stages, in a completely randomized way. In the first stage, corresponding to the evaluation of the biological cycle of egg-adult life, the experiment used 30 couples selected at random from the commercial creation of the laboratory, being accommodated in an individualized way. In the second stage, the viability diagnosis of larva-pupa, larva-adult and pupa-adult was carried out, as well as the sexual ratio of $D$. saccharalis adults. 600 larvae were collected during five weeks (120 units per week). In the third stage, in relation to the evaluations of the experiment to measure the quantity of larvae by couples, it was necessary to adopt four treatments with 10 repetitions each, using an adult insect couple per mating chamber. In all the studied phases, the results highlight that the viability of this host presents rates above $90 \%$. In the adult sex ratio, females are the majority. During the oviposition period, the average amount of larvae obtained per couple was 243 individuals. It is concluded that temperature is an important factor to prolong or decrease the life cycle of $D$. saccharalis. Keywords: Sugar cane borer; Biological control; Production of beneficial insects.
\end{abstract}




\section{Resumen}

La expansión del cultivo de la caña de azúcar atrae a varios insectos plaga, lo que representa un factor limitante para el cultivo de la caña de azúcar en Brasil. El objetivo de la investigación fue analizar aspectos de comportamiento, en laboratorio, del barrenador de la caña de azúcar Diatraea saccharalis como hospedador de Cotesia flavipes. El experimento se desarrolló en tres etapas, de forma completamente aleatoria. En la primera etapa, correspondiente a la evaluación del ciclo biológico de la vida huevo-adulto, el experimento utilizó 30 parejas seleccionadas al azar de la creación comercial del laboratorio, siendo acomodadas de manera individualizada. En la segunda etapa se realizó el diagnóstico de viabilidad de larva-pupa, larva-adulto y pupa-adulto, así como la proporción sexual de los adultos de $D$. saccharalis. Se recolectaron 600 larvas durante cinco semanas (120 unidades por semana). En la tercera etapa, en relación a las evaluaciones del experimento para medir la cantidad de larvas por parejas, fue necesario adoptar cuatro tratamientos con 10 repeticiones cada uno, utilizando una pareja de insectos adultos por cámara de apareamiento. En todas las fases estudiadas, los resultados destacan que la viabilidad de este hospedador presenta tasas superiores al $90 \%$. En la proporción de adultos por sexo, las mujeres son mayoría. Durante el período de oviposición, la cantidad promedio de larvas obtenidas por pareja fue de 243 individuos. Se concluye que la temperatura es un factor importante para prolongar o disminuir el ciclo de vida de $D$. saccharalis.

Palabras clave: Barrenador de la caña de azúcar; Control biológico; Producción de insectos benéficos.

\section{Introdução}

$\mathrm{Na}$ era da agricultura 4.0, a humanidade vivencia os desdobramentos da revolução industrial e da era digital, com a absolvição de seus impactos positivos e nocivos, cujas consequências e influências afetam a vida humana na Terra. Dentre as múltiplas culturas agrícolas, o cultivo de cana-de-açúcar (Saccharum officinarum L.) encontra raízes históricas no âmbito macroeconômico nacional. Da extração de matérias primas ao controle biológico, a engenharia agrícola precisa dar conta de questões ambientais e de aspectos da saúde dos trabalhadores envolvidos nesse processo logístico, extrativista, corporativista e empresarial (Bagliano, 2012).

O controle biológico de pragas representa um dos grandes pilares do Manejo Integrado de Pragas (MIP). Aumentar a produção de predadores e parasitóides é algo desejável em programas de controle biológico (Aquino, 2000, p. 29).

No Brasil, os aparatos tecnológicos e jurídicos tendem a privilegiar ações que reduzam os impactos ambientais, os acidentes de trabalho e a insalubridade do ambiente de trabalho do cultivo de cana-de-açúcar, representando um produto agrícola que tem uma relação intrínseca com o Brasil, inclusive com o Nordeste. Cabe destacar que no cenário internacional, o Brasil é o maior produtor de cana-de-açúcar, com uma produção estimada em 620.832 milhões de toneladas na safra 2018/2019 onde, na região Norte-Nordeste, nesse mesmo período, a produção de cana-de-açúcar foi de aproximadamente 47.707 milhões de toneladas, representando cerca de $8 \%$ de toda a produção nacional (UNICA, 2019). No Estado da Paraíba, por exemplo, essa cultura encontrou condições geográficas e climáticas que favorecem seu manejo (ASPLAN, 2019).

O cultivo da cana-de-açúcar atrai vários insetos-praga como fator limitante ao seu cultivo no Estado da Paraíba. É sabido que há mais de 80 espécies de insetos que tem nesse produto agrícola a sua fonte de alimento, sendo a Diatraea saccharalis (Fabricius, 1794) (Lepidoptera: Crambidae) a principal praga que ataca a cultura (Pinto, et al., 2006).

Além do controle químico das pragas que afetam negativamente a produtividade da cana-de-açúcar, a sustentabilidade dessa cultura perpassa por outros agentes do MIP. O uso contínuo de inseticidas, por exemplo, no combate a D. sacchralis, possui baixa eficácia, pois as lagartas não são atingidas pelo produto no interior dos colmos das plantas (Santos, 2018).

A praga D. saccharali, além de praga da cana-de-açúcar, constitui-se em agente fundamental na produção massal, em laboratório, de se próprio agente de controle biológico, a Cotesia flavipes. Nesse contexto, torna-se imperativo o conhecimento sobre a biologia comportamental (em laboratório) tanto do parasitóide (SILVA, R. B. et al., 2020) quanto do seu hospedeiro.

Esse estudo, portanto, teve como objetivo analisar aspectos comportamentais do hospedeiro D. saccharalis, produzidos em laboratório, com ênfase nos seguintes parâmetros: (i) ciclo biológico ovo-adulto; (ii) viabilidade de larvas e pupas e razão sexual dos adultos; e (iii) quantidade de larvas produzidas por casal de D. saccharalis durante sua vida. 


\section{Material e Métodos}

Os insetos de D. saccharalis foram provenientes do Laboratório de Produção Massal de Controle Biológico (LPMCB) da Associação de Plantadores de Cana da Paraíba (ASPLAN), localizado no município de Mamanguape, Paraíba. Na linha de produção do laboratório, a população de D. saccharalis é alimentada com dieta artificial de Hensley e Hammond (1968) com modificações sugeridas por Parra e Mishfedt (1992) e pela ASPLAN (2019).

A metodologia utilizada no experimento em laboratório para a criação da $D$. saccharalis, fundamenta-se na proposta metodológica do protocolo de Nardin (2004), inoculando os ovos em frascos com dieta artificial, com desenvolvimento das fases imaturas em sala climatizada com temperatura controlada em $29^{\circ} \mathrm{C}$. Após o período de aproximadamente 15 dias, as larvas eclodidas foram transferidas para caixas entomológicas, com dieta artificial, por um período de 10 dias.

Na fase de pupa, as amostras de D. saccharalis foram transferidas para caixas plásticas (45 x $25 \times 6 \mathrm{~cm}) \mathrm{com}$ tampa e papel filtro, permanecendo por seis dias, até os adultos emergirem. Os 80 insetos adultos emergidos (35 fêmeas e 45 machos) foram colocados em câmaras de PVC $(21 \times 10 \mathrm{~cm})$ revestidas com papel sulfite, em sala climatizada a $21^{\circ} \mathrm{C}$, para o acasalamento e oviposição. No dia seguinte, as câmaras foram revisadas e os ovos desinfetados com formol e sulfato de cobre (1\%) e acomodados em saco estéril com algodão umedecido. Após cinco dias foram colocados 250 ovos (já inoculados) em garrafas (contendo a dieta) para eclosão e desenvolvimentos das larvas.

Na primeira etapa, correspondente à avaliação do ciclo biológico de vida ovo-adulto da $D$. saccharalis, utilizou-se 30 casais selecionados aleatoriamente da criação comercial do laboratório, sendo acomodados de modo individualizado, ou seja, um casal por câmara de tubo de PVC $(12$ x $5 \mathrm{~cm})$ revestida com papel sulfite e acomodada em salas climatizadas com três temperaturas distintas: 21,25 e $29^{\circ} \mathrm{C}$, com 10 repetições (Tratamento = temperatura; Repetição = cada casal utilizado).

A segunda etapa avaliou a viabilidade de larva-pupa, larva-adulto e pupa-adulto, bem como a razão sexual dos adultos de D. saccharalis. Foram coletadas 600 larvas durante cinco semanas (gerações distintas), com um total de 120 unidades semanais. Ou seja, o experimento foi realizado em cinco tratamentos (um tratamento por semana), com 120 larvas por tratamento, utilizando material proveniente de lotes da produção comercial do laboratório. Cabe destacar que foram utilizadas 12 larvas por placa Petri, correspondendo a uma repetição por placa, totalizando 10 repetições para cada tratamento. Utilizou-se sala climatizada com temperatura de aproximadamente $28^{\circ} \mathrm{C}$ e fotofase de 12 horas.

A terceira etapa, em relação às avaliações do experimento para mensurar a quantidade de larvas por casais de $D$. saccharalis, executou-se quatro tratamentos com 10 repetições cada um, sendo utilizado um casal adulto de inseto por câmara de acasalamento, totalizando 400 casais de D. saccharalis.

Para a análise dos dados, quanto à normalidade e à homogeneidade de variâncias, utilizou-se o teste de Shapiro-Wilk (Shapiro \& Wilk, 1965) e Barttlet (Snedecor \& Cochran, 1989). Os dados de longevidade de D. saccharalis, em relação as fases de ovo, larva, pupa e insetos adultos (macho e fêmea), foram submetidos à análise de variância, sendo aplicado o modelo de regressão linear, em virtude da ocorrência de diferença significativa $(\mathrm{p} \leq 0,05)$. Utilizou-se o software Cloud Radio Access Networks $\left(\mathrm{CRAN}^{\odot}\right)$.

\section{Resultados}

A partir da Tabela 1, constata-se que, em consequência do aumento da temperatura, a longevidade diminuiu em todas as fases do experimento, (ovo, larva, pupa e adulto). Os resultados obtidos em relação à longevidade da pupa evidenciam uma redução no número de dias em decorrência da temperatura, onde os menores percentuais médios ocorreram entre $25{ }^{\circ} \mathrm{Ce} 29{ }^{\circ} \mathrm{C}$. Acerca dos aspectos referentes ao ciclo da D. saccharalis de ovo a adulto, inclusive das diferenças nos resultados obtidos da razão sexual (fêmea e macho), observa-se que, em decorrência do aumento da temperatura, a longevidade diminuiu em todas as 
fases do experimento (ovo, larva, pupa e adulto). Observa-se, ainda, que o desenvolvimento das larvas é reduzido significativamente na temperatura de $21^{\circ} \mathrm{C}$ quando comparada a temperatura de $29^{\circ} \mathrm{C}$.

Tabela 1 - Longevidade de Diatraea saccharalis para cada fase de desenvolvimento.

\begin{tabular}{ccccccc}
\hline \multirow{2}{*}{ Temperaturas } & \multicolumn{5}{c}{ Dias \pm Erro Padrão } \\
\cline { 2 - 7 } & Ovos & Larvas & Pupas & Adulto & Fêmea & Macho \\
\hline 21 & $13 \pm 0,0 \mathrm{a}$ & $40,7 \pm 0,4 \mathrm{a}$ & $10,3 \pm 0,2 \mathrm{a}$ & $11,15 \pm 0,2 \mathrm{a}$ & $12 \pm 0,3 \mathrm{a}$ & $10,3 \pm 0,2 \mathrm{a}$ \\
25 & $11 \pm 0,0 \mathrm{~b}$ & $15 \pm 0,2 \mathrm{~b}$ & $7,4 \pm 0,2 \mathrm{~b}$ & $5,1 \pm 0,1 \mathrm{~b}$ & $5,7 \pm 0,3 \mathrm{~b}$ & $4,5 \pm 0,2 \mathrm{~b}$ \\
29 & $5 \pm 0,0 \mathrm{c}$ & $10,8 \pm 0,2 \mathrm{c}$ & $6,4 \pm 0,2 \mathrm{c}$ & $4,05 \pm 0,1 \mathrm{c}$ & $4,6 \pm 0,2^{\mathrm{c}}$ & $3,5 \pm 0,2 \mathrm{c}$ \\
\hline CV\% & 0 & 4,4 & 8,6 & 7,05 & 11,13 & 9,51 \\
p-value & 0,0001 & 0,0001 & 0,0001 & 0,0001 & 0,0001 & 0,0001 \\
\hline
\end{tabular}

$\mathrm{abc}=$ Médias seguidas de letras diferentes na coluna diferem pelo teste de Student-Newman-Keuls; CV\%= Coeficiente de variação. \pm EPM= Erro padrão da média. $($ Temperatura $=$ Tratamento). $\mathrm{P}=$ probabilidade. Fonte: Autores.

$\mathrm{Na}$ fase larva/pupa, o percentual médio de viabilidade apresentou percentuais nos lotes dois, três e quatro (Tabela 2), enquanto o menor percentual ocorreu no quinto lote. Para a fase larva/adulto, os lotes 1, 23 e 4 apresentaram os maiores valores, enquanto que o lote, o menor valor. A fase pupa/adulto apresentou percentual médio de $90,70 \%$ de viabilidade: lote $1=92,87 \%$; lote $2=94,91 \%$; lote $3=95,72 \%$; lote $4=96,69 \%$; e, com menor viabilidade, o lote cinco, com apenas $73,32 \%$.

Tabela 2 - Viabilidade de Diatraea saccharalis em cada fase de desenvolvimento e razão sexual.

\begin{tabular}{cccccc}
\hline \multirow{2}{*}{ Lote } & & \multicolumn{2}{c}{ Viabilidade de larva e pupa, e razão sexual } & & \\
\cline { 3 - 7 } & Larva/pupa & Larva/adulto & Pupa/adulto & Fêmea & Macho \\
\hline 1 & $93,33 \pm 2,57 \mathrm{a}$ & $86,67 \pm 4,33 \mathrm{a}$ & $92,87 \pm 3,89 \mathrm{a}$ & $58,43 \pm 6,51 \mathrm{a}$ & $41,57 \pm 6,51 \mathrm{a}$ \\
2 & $91,67 \pm 2,71 \mathrm{a}$ & $87,50 \pm 4,29 \mathrm{a}$ & $94,91 \pm 2,90 \mathrm{a}$ & $53,63 \pm 3,53 \mathrm{a}$ & $46,37 \pm 3,53 \mathrm{a}$ \\
3 & $94,17 \pm 1,93 \mathrm{a}$ & $90,00 \pm 3,90 \mathrm{a}$ & $95,72 \pm 3,90 \mathrm{a}$ & $59,22 \pm 3,22 \mathrm{a}$ & $40,78 \pm 3,22 \mathrm{a}$ \\
4 & $94,17 \pm 2,29 \mathrm{a}$ & $90,83 \pm 2,88 \mathrm{a}$ & $96,69 \pm 2,78 \mathrm{a}$ & $49,66 \pm 6,93 \mathrm{a}$ & $50,34 \pm 6,93 \mathrm{a}$ \\
5 & $78,33 \pm 5,89 \mathrm{~b}$ & $58,33 \pm 7,87 \mathrm{~b}$ & $73,32 \pm 7,95 \mathrm{~b}$ & $56,56 \pm 5,91 \mathrm{a}$ & $43,44 \pm 5,91 \mathrm{a}$ \\
\hline CV\% & 12,08 & 20,27 & 17,47 & 33,93 & 42,31 \\
$p$-value & 0,0001 & 0,0001 & 0,0001 & 0,723 & 0,723 \\
\hline
\end{tabular}

$\mathrm{ab}=$ Médias seguidas de letras diferentes na coluna diferem pelo teste de Student-Newlman-Keuls; CV\%= Coeficiente de variação. $\pm E P M=$ Erro padrão da média. $($ Lote $=$ Tratamento). $\mathrm{p}=$ probabilidade. Fonte: Autores.

\section{Discussão}

A longevidade dos ovos de D. saccharalis, por meio de experimentos que foram realizados em intervalos de 4 ${ }^{\circ} \mathrm{C}$, com temperaturas distintas de 21,25 e $29^{\circ} \mathrm{C}$ (Tabela 1), em função dos dias 13,11 e 5 , respectivamente, demonstram diminuições dos resultados em função do aumento da temperatura. A maior parte das reações enzimáticas são aceleradas com o aumento da temperatura, como o crescimento e desenvolvimento dos insetos, podendo este fator abiótico ter influenciado nesse parâmetro. Deste modo, os resultados obtidos vão de encontro com os estudos de Botelho e Macedo (2002), ao constatarem que a eclosão das larvas ocorre entre 7 e 15 dias, variando significantemente em função da temperatura.

A longevidade de larvas de $D$. saccharalis, com temperatura de $21^{\circ} \mathrm{C}$, apresentou resultados significativos, corroborando com Gallo et al. (2002), ao afirmarem que o ciclo biológico total dura, em geral, mais de 40 dias. A literatura reporta a influência do aumento da temperatura na alteração de parâmetros comportamentais e metabólicos, como a quantidade de lipídios e alterações na síntese energética (Zamani et al., 2006; Garcia-Martin et. Al., 2008; Gingras et al., 2008). Esse aumento de dias no desenvolvimento das larvas deve ser considerado como algo positivo em 
programas de produção massal de parasitóides de $D$. saccharalis. Tal "hibernação" poderá ser manipulada, nesse intervalo estudado, de maneira que a oferta do hospedeiro possa ser feita com base na disponibilidade de parasitóides em laboratório.

Os resultados obtidos em relação à longevidade da pupa evidenciam uma redução no número de dias em decorrência da temperatura, quando comparado com as demais fases como (ovos e larva). Essa redução foi bastante acentuada com o aumento da temperatura (Tabela 1). De fato, em $21^{\circ} \mathrm{C}$, menor temperatura observada, o experimento obteve $80 \%$, apresentando média geral de 10,3 dias. Tais resultados são semelhantes aos obtidos no estudo de Rando et al. (2016), ao obterem uma média de 10 dias de longevidade nos experimentos realizados. As temperaturas de 25 e $29^{\circ} \mathrm{C}$ mostraram que os resultados obtidos em relação a longevidade da pupa apresentaram resultados de médias percentuais abaixo de 9 dias. Esse resultado é próximo ao relato de Lima (2011), o qual relata fase de longevidade próximo a de nove dias.

O ciclo ovo adulto, apresentou valores aproximados, com relação as temperaturas de 21,25 e $29^{\circ} \mathrm{C}$, entre razão sexual (fêmea e macho). Isso mostra claramente que a temperatura atua de forma decisiva na diminuição de dias de longevidade, estando condicionada ao seu aumento, tal como demonstraram Rando et al. (2016). Porém, na fase adulta, os dados destacam que a longevidade em $21^{\circ} \mathrm{C}$ é de 11,15 dias; sendo de 12 dias para fêmeas e 10,3 dias para os machos, semelhantes aos resultados obtidos por Veiga et al. (2013).

Em relação ao número de dias da longevidade da fêmea de $D$. saccharalis, verifica-se que os valores obtidos para a escala de temperatura de $21{ }^{\circ} \mathrm{C}$, apresentou uma média de 12 dias, enquanto para o número de dias da longevidade do macho de D. saccharalis, para a escala de $21^{\circ} \mathrm{C}$, apresentou uma média de 10,3 dias. Isso mostra uma diferença de 1,7 dias em relação a razão sexual (macho e fêmea), onde os melhores índices apontaram para a temperatura de $21^{\circ} \mathrm{C}$. Lima (2011) observa que a mediada que a temperatura aumenta, a quantidade de dias diminui para ambos os sexos. Nos estudos de Rando et al. (2016) relatam que o maior resultado de longevidade dos adultos permanece em torno de nove dias, diferentemente do experimento a $25^{\circ} \mathrm{C}$, com média geral de 5,7 dias, e na temperatura de $29{ }^{\circ} \mathrm{C}$ com média geral em 4,6 dias. Estes resultados apontam para índices abaixo do reportado pela literatura e essas alterações acontecem em decorrência do efeito térmico sobre a taxa metabólica do inseto, uma vez que o metabolismo é suscetível a alterações da temperatura do meio (Irlich et al., 2009). Ou seja, em ambas as temperaturas, os resultados estão abaixo dos índices encontrados na literatura.

Observando os dados da viabilidade em relação aos lotes de larva/adulto, o percentual médio encontrado foi de 82,67\%. Os lotes que apresentaram os maiores percentuais foram os lotes 3 e 4, enquanto que o lote 5 apresentou uma diminuição considerável se comparada aos demais, assemelhando-se aos resultados obtidos nos estudos de Araújo (1987), com 70\% de emergência de adultos da D. saccharalis. Este último, pode estar associado aos eventos apontados por Parra (1999) onde demonstrou que adultos de $D$. saccharalis apresentam considerável redução em sua capacidade de postura em ambientes de baixa umidade ou sem água, podendo ser encontrados maior número de ovos com redução em sua taxa de eclosão.

Esses resultados, juntamente com as informações sobre o parasitóide $C$. flavipes criado em laboratório (Silva et al., 2020), contribuem para o aprimoramento da produção massal de C. flavipes no controle biológico de D. saccharalis.

\section{Conclusão}

Nas condições em que o experimento foi realizado, infere-se que:

1. A Diatraea saccharalis apresenta-se como hospedeiro viável na produção massal de Cotesia flavipes em programas de controle biológico, principalmente em relação à longevidade, razão sexual e quantidade de larva por casal, servindo como norteamento para tomada de decisão em condições de campo;

2. A temperatura é um fator importante para prolongar ou diminuir o ciclo de vida da D. saccharalis;

3. A D. saccharalis apresenta alta viabilidade larval como hospedeira, com média de larvas viáveis de 243 indivíduos por casal, e a população apresenta razão sexual de adultos preponderante para as fêmeas. 
Espera-se que estudos adicionais contemplem o uso de hospedeiro artificial, considerando o alto custo para produzir a dieta na alimentação de $D$. saccharalis.

\section{Agradecimentos}

Aos técnicos do Laboratório de Produção Massal de Controle Biológico (LPMCB) da Associação de Plantadores de Cana da Paraíba (ASPLAN).

\section{Referências}

Aquino, I. S. (2000). O papel da abelha Apis no manjo do bicudo-do-algodoeiro. In: Torres, J. B., Michereff, S. J. (eds.), Desafios no manejo integrado de praga e doenças. 28-38.

Araújo, J. R. (1987). Guia prático para criação da broca da cana-de-açúcar e de seus parasitoides em laboratório, PLANALSUCAR.

Associação De Plantadores De Cana Da Paraíba - ASPLAN. (2019). Balanço final da safra 2018/19 de cana-de-açúcar mostra que a Paraíba manteve média de produção. http://www.asplanpb.com.br.

Bagliano, R. V. Principais organismos utilizados como bioindicadores relatados com uso de avaliadores de danos ambientais. (2012). Revista Meio Ambiente e Sustentabilidade, 2(1), 24-40.

Botelho, P. S. M. \& Macedo, N. Cotesia flavipes para o controle de Diatraea saccharalis. (2002). In: Parra et al. (Ed.). Controle biológico no Brasil: Parasitoides e predadores. Manole, p. 409-425.

Gallo, D. et al. Entomologia agrícola. (2002). (10a ed.), FEALQ.

Garcia-Martin, M., Gámez, M., Torres-Ruiz, A., \& Cabello, T. Functional response of Chelonus oculator (Hymenoptera: Braconidae) to temperature, and its consequences to parasitism. Community Ecology, 9(1), 45-51.

Gingras, D., Dutilleul, P., Boivin, G. Effect of plant structure on searching strategy and searching efficiency of Trichogramma turkestanica. Journal of Insect Science, 8(1), 28.

Hensley, S. D., \& Hammond, A. H. (1968). Laboratory techniques for rearing the sugarcane borer on an artificial diet. Journal of Economic Entomology, 61(6), 1742-1743. http://dx.doi.org/10.1093/jee/61.6.1742.

Irlich, U. M., Terblanche, J. S., Blackburn, T. M., \& Chown, S. L. (2009). Insect rate-temperature relationships: environmental variation and the metabolic theory of ecology. The American Naturalist, 174(6), 819-835. http://www.jstor.org/stable/10.1086/6479042009.

Lima, N. C. A Formação dos Preços do Etanol Hidratado no Mercado Brasileiro de Combustíveis. (2011). 218fs. Tese (Doutorado em Administração). Faculdade de Economia, Administração e Contabilidade. Universidade de São Paulo (USP).

Nardin, R. R. (2004). Protocolo de laboratório para a produção de Cotesia flavipes e Diatraea saccharalis. FEAL.

Parra, J. R. P. \& Mishfedt, L. J. Comparison of artificial diets for rearing the sugarcane borer, In: Anderson, T.E, Leppla, N.C. (eds.), Advances in Insect Rearing for Research and Pest Management. Westview Press, 195-209.

Parra, J. R. P. et al. Efeito da nutrição de adultos e da umidade na fecundidade de Diatraea saccharalis (Fabr.) (Lepidoptera: Crambidae). In: Parra, J.R.P., Milano, P., Consoli, F. L., Zerio, N. G., Haddad, M. L., Efeito da nutrição de adultos e da umidade na fecundidade de Diatraea saccharalis (Fabr.) (Lepidoptera: Crambidae), A. Soc. Entomol. 28(1): 49-57 (1999).

Pinto, A. S., Garcia, J. F., \& Botelho, B. S. M. (2006). Controle Biológico da cana de açúcar. In: Pinto, A. S. et al (Eds). Controle Biológico de Pragas na Prática. PLANALSUCAR, p. 287

Rando, J. S. et al. (2016). Marcação de Diatraea saccharalis (Fabr.) com Diferentes Corantes em Dieta Artificial. Entomo Brasilis, 9(1). https://dialnet.unirioja.es/ descarga/articulo/5470572.pdf.

Santos, R. F. (2018). Qualidade do parasitoide Cotesia flavipes Cameron, 1891 (Hymenoptera: Braconidae) produzido por biofábricas de diferentes regiões do Brasil. 76 f. Tese (Doutorado) - Curso de Agronomia, Faculdade de Ciências Agrárias e Veterinárias- Unesp, Jaboticabal, 2018. Cap. 3. https://repositorio.unesp.br/ handle /11449/157433.

Shapiro, S. S., \& Wilk, M. B. (1965). An analysis of variance test for normality: complete samples. Biometrika, 52(3/4), 591-611.

Silva, R. B. et al. (2020). Aspectos comportamentais do hospedeiro Diatraea saccharalis produzidos em laboratório no litoral paraibano. Research, Society and Development, 10(2), 1-13.

Snedecor, G. W., \& Cochron, (1989). W.G. Statistical methods. (8th.ed.), Iowa State University Press.

União Da Agroindústria Canavieira De São Paulo - ÚNICA. (2019). A indústria da cana-de-açúcar: Etanol, açúcar e bioeletricidade. www.portalunica.com.br/. 
Research, Society and Development, v. 10, n. 5, e1810513507, 2021

(CC BY 4.0) | ISSN 2525-3409 | DOI: http://dx.doi.org/10.33448/rsd-v10i5.13507

Veiga, A. C. P. et al. (2013). Quality control of Cotesia flavipes (Cameron) (Hymenoptera: Braconidae) from different Brazilian biofactories. Biocontrol Science and Technology, 23(6), 665-673.

Zamani, A., Talebi, A., Fathipour, Y., \& Baniameri, V. Temperature-dependent functional response of two aphid parasitoids, Aphidius colemani and Aphidius matricariae (Hymenoptera: Aphidiidae), on the cotton aphid. Journal of Pest Science, 79(4), 183-188. 